\title{
Non-pulmonary manifestations of respiratory syncytial virus infection
}

\author{
Despoina Gkentzi, Gabriel Dimitriou, Ageliki Karatza \\ Department of Paediatrics, Patras Medical School, University of Patras, Patras, Greece \\ Correspondence to: Despoina Gkentzi. Department of Paediatrics, University General Hospital of Patras, Patras 26504, Greece. \\ Email: gkentzid@hotmail.com.
}

Submitted Jun 04, 2018. Accepted for publication Oct 08, 2018.

doi: $10.21037 /$ jtd.2018.10.38

View this article at: http://dx.doi.org/10.21037/jtd.2018.10.38

Respiratory syncytial virus (RSV) infection is an important cause of childhood morbidity from acute lower respiratory tract infections worldwide (1). RSV bronchiolitis is the most common cause of hospitalisation and death due to lower respiratory tract infection in children less than 1 year of age. Approximately $3-10 \%$ of hospitalised infants with RSV bronchiolitis develop acute respiratory failure and need mechanical ventilation in the Paediatric Intensive Care Unit (PICU). RSV is a potentially lethal pathogen in young children, being the third in frequency after Streptococcus pneumoniae and Haemophilus influenzae type b (2). It is a medium-sized RNA virus surrounded by a capsule belonging to the paramyxovirus family, which is multiplied in the epiglottis of the nasopharynx and then dispersed in the epithelium of the small airways and bronchiole (3). Like the other Paramyxoviridae, RSV is also capable of attaching to special receptors on the surface of non-epithelial cells and infect them. Indeed, the virus has been isolated from human myocardial tissue, liver, and cerebrospinal fluid (3). Although most of the research has focused on the mechanics and immunopathology of pulmonary involvement, RSV infection is not exclusively a disease of the lungs. It also significantly affects organs outside the respiratory tract. This particular aspect of RSV disease is underestimated by both paediatricians and respiratory physicians. Clinicians should be aware of the non-pulmonary manifestations of RSV because they can lead to unexpected deterioration in the clinical picture of the patients and may pose significant diagnostic and therapeutic challenges (1). Knowledge of RSV non-pulmonary manifestations is particularly important when treating patients with chronic diseases (4). The non-pulmonary manifestations of severe RSV infections have been reported occurring in the following systems and organs in order of frequency: cardiovascular, central nervous system (CNS), endocrine and liver. Deterioration of patients with chronic diseases involving the above-mentioned systems may be a diagnostic dilemma, as it may be due to worsening of their underlying disease due to the concomitant viral infection or a direct effect of RSV itself.

RSV infection causes neurological complications in approximately $1.2 \%$ of affected infants and children (5). Neurological manifestations include convulsions, central apnoeas, lethargy, feeding and swallowing difficulties, changes in muscle tone, strabismus, cerebrospinal fluid abnormalities, and encephalopathy $(6,7)$. Direct CNS infection and neurological symptoms have been confirmed by the detection of viral RNA and antibodies to RSV in the cerebrospinal fluid. The neurological complications have been attributed to overproduction of cytokines and free radicals. Neuroimaging findings of RSV acute encephalopathy include generalised oedema, abnormal fluid distribution in the brain parenchyma, involving the cerebral cortex, and development of diffuse cerebral atrophy with loss of neurons and their connections $(6,7)$. Central apnoeas and seizures are the most common neurological complications with a frequency, reported though in small studies, up to $40 \%$ of infants requiring mechanical respiratory support $(5,8)$. Two types of convulsions have been described: generalised tonic-clonic and focal seizures. As with RSV encephalopathy, seizures have been attributed to the overproduction of cytokines and free radicals. Hyponatraemia (a common finding in bronchiolitis by RSV) contributes to seizure risk. Direct infestation of the cranial nerves may lead to some rarer clinical manifestations such as strabismus with isotropy (6). 
Cardiovascular system involvement has been reported occurring in $35-45 \%$ of infants requiring mechanical ventilation due to severe RSV infection $(9,10)$. RSV can affect the heart in multiple ways. RSV has been isolated from myocardial tissue biopsies in patients with myocarditis suggesting direct organ invasion. In addition, the virus can cause the development of significant pericardial effusion in some children, which if severe may result in cardiac tamponade. With regards to cardiac rhythm disturbances, in infants with RSV pneumonitis the following have been reported: supraventricular tachycardia, multifocal atrial tachycardia and atrial flutter, ventricular tachycardia with torsades des pointes, ventricular fibrillation and rarely slow junctional escape rhythm and advanced degree atrioventricular block $(11,12)$. However, the mechanisms that lead to cardiovascular compromise remain still largely hypothetical (1). The development of pulmonary hypertension in severe respiratory RSV infections is a potential mechanism of secondary myocardial dysfunction, hypotension and arrhythmias $(13,14)$ Direct heart invasion by RSV is possible, as there are a few reports in which the virus has been isolated from biopsy material (1). However, the involvement of the cardiovascular system is most probably due to immunological mechanisms which seem to account for the majority of the non-pulmonary manifestations of RSV disease and are briefly described later on in this editorial.

As for the endocrine system, anti-diuretic hormone levels are significantly higher in patients with RSV bronchiolitis compared to subjects with upper respiratory tract infection and higher concentrations have been documented in those who require mechanical ventilation, a finding which is not correlated to sodium levels (15). Moreover, intubated children were found to have higher concentrations of prolactin and growth hormone and lower concentrations of leptin and IGF-1 compared to ward inpatients (16). The latter might suggest a relationship between the neuroendocrine response in acute RSV infection and the severity of illness. Bronchiolitis is generally characterised by increased secretion of anti-diuretic hormone and hyper-reninaemia with secondary hyperaldosteronism, which cause water retention, but counter-balance each other with respect to serum sodium (17). However, one should bear in mind that hyponatraemia and secondary hyponatraemic convulsions can be iatrogenic and related to the administration of hypotonic solutions (18). An increase in cortisol levels with a parallel decrease in IL- 12 and IFN- $\gamma$ production, findings compatible with a Th1-type decreased immune response, were found in infants with bronchiolitis. In the subgroup of infants with more severe symptoms, the increase in cortisol levels was more prominent than the reduction of IL-12 and IFN- $\gamma$ production (19). In addition, in those severely affected infants an increase in glucocorticoid beta receptor expression was observed, resulting in decreased in alpha:beta glucocorticoid receptor ratio. Therefore, beta receptors cannot bind to cortisol in order to produce anti-inflammatory activity. This finding appears to be correlated to the severity of the disease and might explain the insensitivity to the administration of steroids (20).

Regarding the hepatic involvement during RSV infection, various mechanisms have been implicated, such as acute hepatitis from the virus itself, high viral load causing dispersal in the systemic circulation, hepatotoxic medications, hepatic congestion or ischaemia secondary to dysfunction of the abdominal circulation (21-23). In addition, during the course of RSV infection, children with congenital heart defects are prone to develop heart failure or pulmonary hypertension which may lead to impaired liver function.

It is important to mention here that the underlying mechanisms and pathophysiology of the non-pulmonary manifestations of RSV diseases are still not well defined. It is unlikely that co-infection with bacteria would be responsible for the majority of the non-pulmonary manifestations in children with RSV bronchiolitis. It is estimated that approximately $1 \%$ of the cases have a concomitant bacterial co-infection, such as urinary tract infection $(24,25)$. In neonates and infants hospitalised in PICU with RSV isolated from the nasopharynx, viral RNA was also detected in peripheral blood by RT-PCR (26). The isolation from blood however, does not guarantee that $\mathrm{RSV}$ is viable and could well reflect RSV genome presence following phagocytosis from neutrophils or monocytes. It is important to mention here that in mice although there is no evidence of RSV involvement in organs other than lungs, viral replication was shown in the peripheral blood of infected mice (27). In humans, most extravascular manifestations are believed to be the result of the release of inflammatory mediators, such as cytokines and chemokines. The lung pathology developed in RSV-infected individuals is characterised by an exacerbated proinflammatory and unbalanced Th2-type immune response. The immune response is mediated by infected epithelial cells of the respiratory system and alveolar macrophages. Host genetic factors can lead to excessive immune reaction outside the 
lungs. In addition, the virus like the other Paramyxoviridae can also affect non-epithelial cells. This mechanism could partially explain some of the non-pulmonary manifestations of RSV infection where direct invasion of the relevant organs has been documented.

Further research is required to clarify other various areas in the field of non-pulmonary manifestations of RSV infection. First of all, whether medications such as caffeine would be useful in the prevention of mechanical support in severely affected infants (28). Moreover, we need to investigate the long-term neurological consequences in infants with CNS complications and particularly in those with abnormal neuroimaging findings. In addition, the non -pulmonary manifestations of RSV infection in the immunocompromised individuals as well as adults with comorbidities are not well described and understood. Most importantly, there is a gap of knowledge in the frequency of cardiac and CNS involvement in infants with mild infections in the community or in those hospitalised in the general paediatric ward (11). This area has not been thoroughly investigated, and given the disease burden outside the PICU merits further clarification. Finally, we anticipate with interest data on the impact of the preventative strategies targeting RSV bronchiolitis, such as monoclonal antibodies and vaccines, on the epidemiology of the RSV disease and subsequently the non-pulmonary manifestations.

In conclusion, non-pulmonary manifestations are not uncommon in children who are hospitalised for severe RSV bronchiolitis. These patients need monitoring of heart rate and blood pressure to avoid serious complications. Serum sodium levels should be checked daily in those receiving parenteral infusions and maintaining strict fluid balance is essential to prevent hyponatraemia. The latter is quite common and can lead to convulsions. Potentially fatal non-pulmonary complications of severe RSV infection include central apnoea, epileptic seizures, ventricular tachycardia or fibrillation, atrioventricular block and myocarditis, conditions that should be timely detected with proper monitoring. Various areas in the field need further investigation. The huge disease burden outside the PICU highlights the need to describe more accurately the nonpulmonary manifestations of milder RSV disease which are often underestimated by the clinicians.

\section{Acknowledgements}

None.

\section{Footnote}

Conflicts of Interest: The authors have no conflicts of interest to declare.

\section{References}

1. Eisenhut M. Extrapulmonary manifestations of severe respiratory syncytial virus infection--a systematic review. Critical Care 2006;10:R107.

2. Hall CB. Respiratory syncytial virus in young children. Lancet 2010;375:1500-2.

3. Thorburn K, Hart CA. Think outside the box: extrapulmonary manifestations of severe respiratory syncytial virus infection. Critical Care 2006;10:159.

4. Carrat F, Leruez-Ville M, Tonnellier M, et al. A virologic survey of patients admitted to a critical care unit for acute cardiorespiratory failure. Intensive Care Med 2006;32:156-9.

5. Sweetman LL, Ng YT, Butler IJ, et al. Neurologic complications associated with respiratory syncytial virus. Pediatr Neurol 2005;32:307-10

6. Bohmwald K, Espinoza JA, Rey-Jurado E, et al. Human Respiratory Syncytial Virus: Infection and Pathology. Semin Respir Crit Care Med 2016;37:522-37.

7. Morichi S, Kawashima H, Ioi H, et al. Classification of acute encephalopathy in respiratory syncytial virus infection. J Infect Chemother 2011;17:776-81.

8. Antonucci R, Fanos V. Acute encephalopathy associated with respiratory syncytial virus infections in childhood. A literature review. Minerva Pediatr 2005;57:137-42.

9. Checchia PA, Appel HJ, Kahn S, et al. Myocardial injury in children with respiratory syncytial virus infection. Pediatr Crit Care Med 2000;1:146-50.

10. Eisenhut M, Sidaras D, Johnson R, et al. Cardiac troponin $\mathrm{T}$ levels and myocardial involvement in children with severe respiratory syncytial virus lung disease. Acta Paediatr 2004;93:887-90.

11. Esposito S, Salice P, Bosis S, et al. Altered cardiac rhythm in infants with bronchiolitis and respiratory syncytial virus infection. BMC Infect Dis 2010;10:305.

12. Karatza AA, Kiaffas M, Rammos S. Complete heart block complicating the acute phase of respiratory syncytial virus bronchiolitis. Pediatr Pulmonol 2017;52:E61-3.

13. Konstantinides S, Geibel A, Olschewski M, et al. The importance of cardiac troponins $\mathrm{I}$ and $\mathrm{T}$ in risk stratification of patients with acute pulmonary embolism. 
Circulation 2002;106:1263-8.

14. Sreeram N, Watson JG, Hunter S. Cardiovascular effects of acute bronchiolitis. Acta Paediatr Scand 1991;80:133-6.

15. van Steensel-Moll HA, Hazelzet JA, van der Voort E, et al. Excessive secretion of antidiuretic hormone in infections with respiratory syncytial virus. Arch Dis Child 1990;65:1237-9.

16. Tasker RC, Roe MF, Bloxham DM, et al. The neuroendocrine stress response and severity of acute respiratory syncytial virus bronchiolitis in infancy. Intensive Care Med 2004;30:2257-62.

17. Gozal D, Colin AA, Jaffe M, et al. Water, electrolyte, and endocrine homeostasis in infants with bronchiolitis. Pediatr Res 1990;27:204-9.

18. Hanna S, Tibby SM, Durward A, et al. Incidence of hyponatraemia and hyponatraemic seizures in severe respiratory syncytial virus bronchiolitis. Acta Paediatr 2003;92:430-4.

19. Pinto RA, Arredondo SM, Bono MR, et al. T helper 1/T helper 2 cytokine imbalance in respiratory syncytial virus infection is associated with increased endogenous plasma cortisol. Pediatrics 2006;117:e878-86.

20. Diaz PV, Pinto RA, Mamani R, et al. Increased expression of the glucocorticoid receptor beta in infants with RSV bronchiolitis. Pediatrics 2012;130:e804-11.

21. Oh JS, Choi JS, Lee YH, et al. The Relationships between Respiratory Virus Infection and Aminotransferase

Cite this article as: Gkentzi D, Dimitriou G, Karatza A. Non-pulmonary manifestations of respiratory syncytial virus infection. J Thorac Dis 2018;10(Suppl 33):S3815-S3818. doi: $10.21037 /$ jtd.2018.10.38 in Children. Pediatr Gastroenterol Hepatol Nutr 2016;19:243-50.

22. Thorburn K, Fulton C, King C, et al. Transaminase levels reflect disease severity in children ventilated for respiratory syncytial virus (RSV) bronchiolitis. Sci Rep 2018;8:1803.

23. Kirin BK, Topić RZ, Dodig S. Hepatitis during respiratory syncytial virus infection--a case report. Biochem Med (Zagreb) 2013;23:112-6.

24. Randolph AG, Reder L, Englund JA. Risk of bacterial infection in previously healthy respiratory syncytial virusinfected young children admitted to the intensive care unit. Pediatr Infect Dis J 2004;23:990-4.

25. Kaluarachchi D, Kaldas V, Roques E, et al. Comparison of urinary tract infection rates among 2- to 12-month-old febrile infants with RSV infections using 1999 and 2011 AAP diagnostic criteria. Clin Pediatr (Phila) 2014;53:742-6.

26. Rohwedder A, Keminer O, Forster J, et al. Detection of respiratory syncytial virus RNA in blood of neonates by polymerase chain reaction. J Med Virol 1998;54:320-7.

27. Torres JP, Gomez AM, Khokhar S, et al. Respiratory Syncytial Virus (RSV) RNA loads in peripheral blood correlates with disease severity in mice. Respir Res 2010;11:125.

28. Alansari K, Toaimah FH, Khalafalla H, et al. Caffeine for the Treatment of Apnea in Bronchiolitis: A Randomized Trial. J Pediatr 2016;177:204-211.e3. 